Rapid quantitation of flecainide in human plasma for therapeutic drug monitoring using liquid chromatography and time-of-flight mass-spectrometry.

Mohammed Rahman ${ }^{1,2}$, Lewis Couchman $\mathrm{PhD}^{1,3}$, Valentyna Povstyan ${ }^{1}$, Verity Bainbridge ${ }^{1}$, Karin Kipper $\mathrm{PhD}^{1,4}$, Atholl Johnston $\mathrm{PhD}^{1,5}$, David Holt DSc (Med) ${ }^{1}$

${ }^{1}$ Analytical Services International (ASI), St George's University of London, Cranmer Terrace, London SW17 0RE, UK.

${ }^{2}$ Department of Chemistry, University of Surrey, Guildford, GU2 7XH, UK.

${ }^{3}$ Pharmaceutical Sciences Clinical Academic Group, King’s College London, SE1 9NH, UK.

${ }^{4}$ Institute of Chemistry, University of Tartu, 14a Ravila Street, 50411 Tartu, Estonia.

${ }^{5}$ Clinical Pharmacology, William Harvey Research Institute, Barts and The London School of Medicine and Dentistry, Queen Mary University of London, London, EC1M 6BQ, UK

Running head: Rapid flecainide TDM

Address for correspondence:

Analytical Services International,

St. George's - University of London,

Cranmer Terrace, London SW17 0RE.

Tel : +44 (0)20 87252338 
Fax : +44 (0)20 87679687

\section{Email : lewis.couchman@bioanalytics.co.uk}

\section{Abstract}

Background: Measurement of flecainide is useful to optimize dosage and minimise the risks of toxicity. Further, there is a need for urgent sample analysis when flecainide is used in transplacental therapy for foetal tachycardia. To this end, we have developed and validated a rapid assay for the measurement of flecainide in human plasma or serum, using a small sample volume $(50 \mu \mathrm{L})$.

Method: Following a simple deproteination with zinc sulphate and methanol, prepared samples were injected onto a short $(30 \mathrm{~mm})$ analytical column, and eluted using a rapid gradient elution. Detection was carried out using time-of-flight mass-spectrometry. Flecainide was quantified using flecainide- $\mathrm{D}_{4}$ as internal standard, with both compounds extracted from the total-ion chromatogram using a \pm 5 ppm extraction window based on theoretical $\mathrm{m} / \mathrm{z}$ values for the protonated ions.

Results: The assay was linear over a putative therapeutic range (100 - 1,000 $\mu \mathrm{g} / \mathrm{L})$. Betweenand within-assay precision and accuracy were $<4.6 \%$ and $94.8-110.0 \%$, respectively. Matrix effects were minimal, and were compensated for by flecainide- $\mathrm{D}_{4}$. There were no effects due to haemolysis or lipaemia, and no carryover was apparent. Total analysis time was just 1.2 minutes (72 s).

Conclusions: We have developed and validated a rapid method for the analysis of flecainide. The method is particularly suited to flecainide TDM, when analysing samples from mothers receiving flecainide for the treatment of foetal tachycardia.

\section{Keywords}


LC-TOF-MS; mass-spectrometry; liquid chromatography; high-throughput; flecainide; paediatric

\section{Conflicts of Interest}

None to declare. 


\section{Introduction}

Flecainide is a class 1C (Vaughan-Williams) anti-arrhythmic agent used in the treatment of both atrial and ventricular tachyarrhythmias. ${ }^{[1]}$ It is also highly effective as an oral maternal transplacental therapy in foetal tachycardia $(\mathrm{FT}),{ }^{[1,2]}$ though the risk of pro-arrhythmic effects in some patients have limited its use. ${ }^{[3]}$ The clinical efficacy and the risk of adverse events are associated with plasma or serum flecainide concentrations, which do not always correlate well with the prescribed dose due to variability in renal and hepatic clearance. Adverse events have been associated with flecainide concentrations greater than $1,000 \mu \mathrm{g} / \mathrm{L},{ }^{[4,5]}$ and case reports are available of severe iatrogenic flecainide toxicity in neonates associated with dosing errors leading to elevated flecainide concentrations. ${ }^{[6,7]}$ The lower concentration limit associated with anti-arrhythmic effect is typically defined as $200 \mu \mathrm{g} / \mathrm{L},{ }^{[8]}$ though more recently a limit of $300 \mu \mathrm{g} / \mathrm{L}$ has been suggested for patients with tachyarrhythmia. ${ }^{[9]}$ Monitoring plasma or serum concentrations of the drug is therefore an important part of the management of patients receiving flecainide anti-arrhythmic therapy, to guide dosage adjustments to maximise clinical efficacy with minimal risk of adverse events. ${ }^{[8]}$

To maximise the clinical benefits of therapeutic drug monitoring (TDM) analytical results should be available as soon as possible to allow rapid dose adjustments, if necessary. However, such assays are often considered 'specialist' tests, and are not routinely available in many settings, certainly as urgent tests. Even within specialist laboratories using liquid chromatography, lengthy sample preparation and chromatographic methods for flecainide have been used, ${ }^{[10-13]}$ and are not suited to urgent analyses. In this paper, we report the validation of a simple, rapid, liquid chromatography time-of-flight mass-spectrometry (LCTOF-MS) method for total plasma flecainide, suitable for use for TDM, and for urgent analyses relevant during the treatment of FT. 


\section{Materials and methods}

\section{Chemicals and reagents}

( \pm )-Flecainide acetate, zinc sulfate heptahydrate, isopropanol, ammonium formate, formic acid (LC-MS grade) and acetic acid were from Sigma Aldrich (Poole, UK). Methanol (LCMS grade) was from Rathburn (Walkerburn, Scotland). ( \pm )-Flecainide- $\mathrm{D}_{4}$ acetate was from Alsachim (Strasbourg, France). Water was deionised, prepared in-house $(<1.0 \mu \mathrm{S} / \mathrm{cm})$. Analyte-free human plasma (lithium heparin and EDTA) and analyte-free human serum from individual donors were from Biological Speciality Corporation (Colmar, PA, USA). Calibrant and reference ion stock solutions for the TOF-MS were from Agilent Technologies (Santa Clara, CA, USA) and were diluted according to the manufacturer's instructions prior to use. External quality assessment (EQA) samples were from the LGC Therapeutic Drugs scheme (LGC, Lancashire, UK).

\section{Chromatography}

A 1290 binary LC pump was used with a 1290 Infinity II ${ }^{\mathrm{TM}}$ autosampler (Agilent Technologies). Analysis was performed using a Raptor ${ }^{\mathrm{TM}}$ biphenyl column (30 x $2.1 \mathrm{~mm}, 2.7$ $\mu \mathrm{m}$ total particle size; Thames Restek, Saunderton, UK) held at $40{ }^{\circ} \mathrm{C}$ in a column oven (MCT 1290, Agilent Technologies). System eluents were as follows: eluent A, $10 \mathrm{mmol} / \mathrm{L}$ ammonium formate in $0.01 \%(v / v)$ formic acid in deionised water; eluent $\mathrm{B}, 10 \mathrm{mmol} / \mathrm{L}$ ammonium formate in $0.01 \%(v / v)$ formic acid in methanol. Eluent B was also used as the autosampler needle wash solvent. The piston seal wash was $50 \%(v / v)$ isopropanol in deionised water. Eluent flow was diverted to waste for the first $12 \mathrm{~s}$ of each analysis. Total analysis time was 72 seconds (1.2 $\mathrm{min})$. 


\section{Mass-spectrometry}

A 6230 time-of-flight mass-spectrometer (Agilent Technologies) was used with a dual-spray electrospray ionisation source (Jet Stream ${ }^{\mathrm{TM}}$, Agilent Technologies). Source conditions were: gas (high-purity nitrogen) temperature $325^{\circ} \mathrm{C}$, gas flow-rate $12 \mathrm{~L} / \mathrm{min}$, nebulizer gas pressure $60 \mathrm{psig}$, sheath gas temperature $350{ }^{\circ} \mathrm{C}$, sheath gas flow-rate $12 \mathrm{~L} / \mathrm{min}$, capillary voltage 3,750 V. Instrument control and data acquisition were performed using MassHunter ${ }^{\mathrm{TM}}$ (version B.08.00, Agilent Technologies). TOF-MS data were acquired from 50-1,000 m/z. Acquisition rate was 5 scans per second (2,639 transients/spectrum). Mass calibration was carried out daily using calibration solution stored on the instrument, and reference ion solution (containing reference ions at $\mathrm{m} / z, 121.0509$ and 922.0098) was delivered throughout the analysis via the second nebuliser of the dual spray source at a constant flow rate using a 1260 isocratic pump (Agilent Technologies), flowing at $1.00 \mathrm{~mL} / \mathrm{min}$ with a 1:100 flow splitter $(10 \mu \mathrm{L} / \mathrm{min}$ flow-rate at-source).

For data processing, flecainide and flecainide- $\mathrm{D}_{4}$ (empirical formulae $\mathrm{C}_{17} \mathrm{H}_{20} \mathrm{~F}_{6} \mathrm{~N}_{2} \mathrm{O}_{3}$ and $\mathrm{C}_{17} \mathrm{H}_{16} \mathrm{D}_{4} \mathrm{~F}_{6} \mathrm{~N}_{2} \mathrm{O}_{3}$, respectively) chromatograms were extracted as protonated ions using \pm 5 ppm extraction windows $\left(\mathrm{m} / \mathrm{z}, 415.1451\right.$ and 419.1702 for flecainide and flecainide- $\mathrm{D}_{4}$, respectively). The naturally occurring ${ }^{13} \mathrm{C}$ isotope of flecainide $(\mathrm{m} / \mathrm{z}, 416.1490)$ was used as a qualifier ion. The acceptance range for the extracted flecainide- ${ }^{13} \mathrm{C}$ peak area was 13.5 $22.5 \%$ that of the flecainide peak area.

\section{Sample preparation}

Samples, calibrators, and control solutions were thawed, if necessary, and mixed at room temperature by inversion. Samples, calibrators, and controls $(50 \mu \mathrm{L})$ and working internal standard solution $(50 \mu \mathrm{L})$ were pipetted into $1.5 \mathrm{~mL}$ micro-centrifuge tubes. Freshly prepared 
precipitation reagent $(0.2 \mathrm{~mol} / \mathrm{L}$ aqueous zinc sulfate solution in methanol, $2+8, v / v)$ was then added. The tubes were capped and vortex mixed (60 s), prior to centrifugation $(13,200$ rpm, 5 min). Supernatants were transferred with disposable pipettes to reduced-volume autosampler vials, and capped for LC-TOF-MS analysis. The LC auto-sampler tray was cooled to $10{ }^{\circ} \mathrm{C}$, and $1 \mu \mathrm{L}$ was injected for analysis.

\section{Calibrators, internal quality control (IQC), and internal standard solutions}

Calibrator and IQC stock solutions (both $100 \mathrm{mg} / \mathrm{L}$ flecainide) were prepared in methanol, and were stored frozen $\left(-18\right.$ to $\left.-20^{\circ} \mathrm{C}\right)$. Calibrators were prepared by appropriate dilution of the calibrator stock solution with analyte-free human plasma to contain 100, 250, 500, 750, 1,000 and 1,500 $\mu \mathrm{g} / \mathrm{L}$ flecainide. IQC solutions were similarly prepared by appropriate dilution of the IQC stock solution, prepared from a separate weighing, with analyte-free human plasma to contain 200, 400 and $800 \mu \mathrm{g} / \mathrm{L}$ flecainide. Once prepared, calibrators and IQC solutions were allowed to equilibrate overnight $\left(2\right.$ to $\left.8{ }^{\circ} \mathrm{C}\right)$ and were then aliquoted into approximately $250 \mu \mathrm{L}$ portions in $1.5 \mathrm{~mL}$ micro-centrifuge tubes, which were stored frozen (18 to $-20{ }^{\circ} \mathrm{C}$ ) until required for analysis.

Flecainide- $\mathrm{D}_{4}$ stock solution (approximately $100 \mathrm{mg} / \mathrm{L}$ ) was prepared in methanol, and stored frozen $\left(-18\right.$ to $-20{ }^{\circ} \mathrm{C}$ ). Working internal standard solution (approximately $500 \mu \mathrm{g} / \mathrm{L}$ ) was prepared by $1+199(v / v)$ dilution of the flecainide- $\mathrm{D}_{4}$ stock solution with $0.5 \%(v / v)$ aqueous acetic acid. Working internal standard solution was stored at 2 to $8{ }^{\circ} \mathrm{C}$. In addition, a system suitability test (SST) solution was prepared in methanol containing flecainide $(50 \mu \mathrm{g} / \mathrm{L})$. This was stored at 2 to $8^{\circ} \mathrm{C}$, and portions were transferred to auto-sampler vials for direct analysis prior to starting batch analysis, in order to check instrument performance. 


\section{Method validation}

Within-batch precision (\% RSD) and accuracy were assessed by replicate analysis $(\mathrm{N}=10)$ of the two IQC solutions, plus the lower limit of quantitation (LLoQ) and upper limit of quantitation (ULoQ) calibrators $(100 \mu \mathrm{g} / \mathrm{L}$ and 1,500 $\mu \mathrm{g} / \mathrm{L}$, respectively). Between-batch precision and accuracy were assessed using the mean result (from at least duplicate analyses within each batch) given by the two IQC samples, and by the LLoQ and ULoQ calibrators on separate days ( $\mathrm{N}=10$ batches) over a three-week period.

Carryover was assessed using a high-concentration sample prepared in analyte-free plasma containing 5,000 $\mu \mathrm{g} / \mathrm{L}$ flecainide (greater than three times the ULoQ calibrator). This sample was prepared and analysed bracketed by triplicate analyte-free plasma samples, and carryover was assessed by comparison of the flecainide peak areas in the samples before and after the high-concentration samples.

Linearity was assessed by serial dilutions (each $1+1, v / v$ with analyte-free plasma) of the ULoQ calibrator. Each diluted sample was analysed in triplicate within a single batch, and the accuracy (observed vs expected concentration) and precision of each dilution was calculated. Autosampler stability $\left(10^{\circ} \mathrm{C}\right)$ was assessed by preparation of a calibration curve, which was analysed (i) immediately, (ii) after 24 hours, and (iii) after 48 hours in the autosampler tray. Additional stability of flecainide in human plasma was assessed using the three IQC solutions. IQC solutions were prepared and stored in various conditions - at ambient temperature for one week, refrigerated $\left(2\right.$ to $\left.8^{\circ} \mathrm{C}\right)$ for one week, after three freeze-thaw cycles, and after frozen long-term storage $\left(-15\right.$ to $\left.-25^{\circ} \mathrm{C}\right)$ for 9 months. Stability samples were analysed against freshly-prepared calibrators stored frozen $\left(-15\right.$ to $\left.-25^{\circ} \mathrm{C}\right)$ and thawed only once prior to analysis. 
Matrix effects and analyte recovery were assessed quantitatively using pre- and postextraction spiking experiments as described by Matuzewski et al., at 200 and $500 \mu \mathrm{g} / \mathrm{L}$ for both flecainide and flecainide- $\mathrm{D}_{4} \cdot{ }^{[14]}$ Matrix effects were assessed using six independent analyte-free matrices (two EDTA plasma, two lithium heparin plasma and two serum matrices). The influence of lipaemia and haemolysis were assessed using mixing experiments. ${ }^{[15]}$ Briefly, lipaemic and haemolysed samples were prepared containing flecainide at 1,500 $\mu \mathrm{g} / \mathrm{L}$. These samples were mixed in different proportions with analyte-free 'normal' plasma (i.e. not lipaemic or haemolysed) containing flecainide at $100 \mu \mathrm{g} / \mathrm{L}$ to produce five-point curves. The mean measured concentrations were compared to the expected concentrations to assess any bias due to these factors.

\section{Samples}

Patient plasma samples $(\mathrm{N}=60)$ and EQA samples $(\mathrm{N}=8)$ that had been submitted for flecainide TDM were extracted using the method described here and analysed by LC-TOFMS. The results were compared (Analyse-It for Microsoft Excel, 2010 version) with those obtained using a previously validated manual liquid-liquid extraction, liquid chromatographytandem mass-spectrometry (LC-MS/MS) method (see Details, Supplemental Digital Content 1, which briefly describes the LC-MS/MS method). All LC-MS/MS analyses were carried out within 3 days of the sample arriving in the laboratory, and subsequent LC-TOF-MS analysis within 80 days. Samples were stored at 2 to $8{ }^{\circ} \mathrm{C}$ between analyses.

\section{Results}

Example chromatograms for flecainide, flecainide- ${ }^{13} \mathrm{C}$ (qualifier ion) and flecainide- $\mathrm{D}_{4}$ are shown in Figure 1. Typical retention times were 43.7 and $43.5 \mathrm{~s}$ for flecainide and flecainide- 
$\mathrm{D}_{4}$, respectively, and the total analysis time was just 72 seconds. Calibration was linear (typical $\mathrm{R}^{2}>0.98$ ) over the range $100-1,500 \mu \mathrm{g} / \mathrm{L}$ (see Table, Supplemental Digital Content 2, which summarises the serial dilution linearity test). Within-batch and between-batch precision (\% RSD) and accuracy (\% nominal concentrations) were $<4.6 \%$ and $94.8-110.0$ $\%$, respectively (Table 1$)$.

No carryover was observed (no peaks in the analyte-free samples analysed directly after triplicate analysis of the high-concentration sample), and prepared samples were stable when stored on the autosampler for $48 \mathrm{~h}\left(10^{\circ} \mathrm{C}\right)$. Flecainide was stable in plasma under all conditions tested (Table 2).

The mean (SD) overall process efficiency for flecainide was 111.7 (4.22) \%, and the mean (SD) matrix effect was 104.2 (4.24) \%, suggesting some ionisation enhancement. However, the mean (SD) relative process efficiency and matrix effect, based on flecainide peak areas relative to flecainide- $\mathrm{D}_{4}$ peak areas, was $99.7(0.33) \%$ and $99.8(0.53) \%$, respectively. There were no differences in overall process efficiency or observed matrix effects between EDTA plasma, lithium heparin plasma, and serum matrices. There was no observed bias as a result of haemolysis or lipaemia (see Table, Supplemental Digital Content 3, which displays the results of the mixing experiments).

Comparison of results $(\mathrm{N}=62$, inclusive of patient and EQA samples) acquired via the rapid LC-TOF-MS method with an existing LC-MS/MS method using liquid-liquid extraction gave excellent agreement $\left(y=0.9895 x+16.679 ; R^{2}=0.9905\right)$. The mean $(S D)$ bias between the methods was $12.3(30.7) \mu \mathrm{g} / \mathrm{L}[3.7(6.6) \%] .6$ samples (5 patient samples and 1 EQA sample) contained flecainide below $100 \mu \mathrm{g} / \mathrm{L}$ (Figure 2). 


\section{Discussion}

We have developed and validated a simple, rapid, method for the quantitation of total flecainide in human plasma and serum, ideally suited to use for flecainide TDM. In particular, the method is suitable for rapid turn-around of samples from patients receiving flecainide for in utero treatment of FT, or from neonates, infants or children with tachyarrhythmias. With simplified sample preparation and rapid batch analysis using the described LC-TOF-MS method, urgent analyses can be carried out within approximately one hour of sample receipt.

The method uses LC-TOF-MS, which is uncommon for TDM analyses, but which does offer some advantages over other, more common, techniques such as LC-MS/MS. The assay demonstrated linear calibration for flecainide analysis across the required concentration range, with no evidence of peak saturation or mass inaccuracy at higher concentrations. The sensitivity of the method was comparable to that of our previously validated method using LC-MS/MS, without requiring additional sample volume (which often is not possible for samples from neonates), and despite the much simpler sample preparation method. The two methods compared very well with one another, and the LC-TOF-MS method performed well for retrospective EQA sample analysis.

Whereas LC-MS/MS uses product ions and product ion ratios for sensitivity and selectivity, LC-TOF-MS relies on high-resolution data acquisition. In this method, we also extracted the $m / z$ for the naturally occurring ${ }^{13} \mathrm{C}$ isotope of flecainide for use as a pseudo-qualifier ion. An alternative approach (in the absence of a quadrupole for fragmentation) would be to use a high source voltage to induce in-source fragmentation, and then to monitor a precursor and product ion (and ion ratio). 
For rapid methods in particular, there are additional benefits of using LC-TOF-MS, rather than targeted LC-MS/MS. Firstly, although the method described in this study is for a single analyte and IS, multiplexed analysis, i.e. the simultaneous analysis of multiple drugs/metabolites, is perhaps better suited to LC-TOF-MS than LC-MS/MS. With LCMS/MS, each additional compound requires an apportionment of the acquisition cycle-time (often referred to as 'dwell time'). Ideally, each additional compound would require more than one transition to allow product ion ratio calculations, and a (co-eluting) internal standard. ${ }^{[16]}$ Therefore, for each additional concurrent ion transition in LC-MS/MS, one must consider dwell-time in relation to the number of data points across a chromatographic peak for accurate quantitation - this may limit the number of simultaneous analytes which can be measured in a short chromatographic analysis. With LC-TOF-MS, the cycle-time is set by the TOF-MS scan-rate, irrespective of the number of components to be later extracted from the acquired MS data.

A coincidental advantage of such rapid analysis is that of data file size. High-resolution MS data files are much larger than LC-MS/MS data files, but are directly proportional to the analysis time - by reducing the analysis time significantly, data storage and transfer becomes quicker and cheaper.

In this study, we have reported a novel method for flecainide TDM. The method is significantly faster than previously published methods, e.g. more than 6 minutes faster per injection than the method recently reported by Mano et al. ${ }^{[17]}$ Further, we have demonstrated for the first time the application of LC-TOF-MS for flecainide quantitation, and have highlighted the possible benefits of this approach for other analytes for which TDM is indicated. 


\section{References}

1. Till JA, Shinebourne EA, Rowland E, Ward DE, Bhamra R, Haga P, Johnston A and Holt DW Paediatric use of flecainide in supraventricular tachycardia: clinical efficacy and pharmacokinetics Br Heart J. 1989;62:133-139.

2. Ekiz A, Kaya B, Bornaun H, Acar DK, Avci ME, Bestel A and Yildirim G Flecainide as first-line treatment for fetal supraventricular tachycardia J Matern Fetal Neonatal Med. 2018;31:407-412.

3. Oudijk MA, Ruskamp JM, Ambachtsheer BE, Ververs TF, Stoutenbeek P, Visser GH and Meijboom EJ Drug treatment of fetal tachycardias Paediatr Drugs. 2002;4:49-63.

4. Morganroth $\mathrm{J}$ and Horowitz LN Flecainide: its proarrhythmic effect and expected changes on the surface electrocardiogram Am J Cardiol. 1984;53:89b-94b.

5. $\quad$ Roden DM and Woosley RL Drug therapy. Flecainide $N$ Engl J Med. 1986;315:36-41.

6. Jang DH, Hoffman RS and Nelson LS A case of near-fatal flecainide overdose in a neonate successfully treated with sodium bicarbonate J Emerg Med. 2013;44:781-783.

7. Karmegaraj B, Menon D, Prabhu MA and Vaidyanathan B Flecainide toxicity in a preterm neonate with permanent junctional reciprocating tachycardia Ann Pediatr Cardiol. 2017;10:288-292.

8. Conard GJ, Cronheim GE and Klempt HW Relationship between plasma concentrations and suppression of ventricular extrasystoles by flecainide acetate (R818), a new antiarrhythmic, in patients Arzneimittelforschung. 1982;32:155-159.

9. Homma M, Kuga K, Doki K, Katori K, Yamaguchi I, Sugibayashi K and Kohda Y Assessment of serum flecainide trough levels in patients with tachyarrhythmia J Pharm Pharmacol. 2005;57:47-51.

10. Bhamra RK, Flanagan RJ and Holt DW High-performance liquid chromatographic method for the measurement of mexiletine and flecainide in blood plasma or serum $J$ Chromatogr. 1984;307:439-444. 
11. Breindahl $\mathrm{T}$ Therapeutic drug monitoring of flecainide in serum using highperformance liquid chromatography and electrospray mass spectrometry $J$ Chromatogr B Biomed Sci Appl. 2000;746:249-254.

12. Nakagawa R, Homma M, Kuga K, Yamaguchi I, Kobayashi D, Morimoto Y and Kohda Y High performance liquid chromatography for routine monitoring of serum flecainide J Pharm Biomed Anal. 2002;30:171-174.

13. Doki K, Homma M, Kuga K, Watanabe S, Yamaguchi I and Kohda Y Simultaneous determination of serum flecainide and its metabolites by using high performance liquid chromatography J Pharm Biomed Anal. 2004;35:1307-1312.

14. Matuszewski BK, Constanzer ML and Chavez-Eng CM Strategies for the assessment of matrix effect in quantitative bioanalytical methods based on HPLC-MS/MS Anal Chem. 2003;75:3019-3030.

15. Couchman L, Subramaniam K, Fisher DS, Belsey SL, Handley SA and Flanagan RJ Automated Analysis of Clozapine and Norclozapine in Human Plasma Using Novel Extraction Plate Technology and Flow-Injection Tandem Mass Spectrometry Ther Drug Monit. 2016;38:42-49.

16. Couchman L, Fisher DS, Subramaniam K, Handley SA, Boughtflower RJ, Benton CM and Flanagan RJ Ultra-fast LC-MS/MS in therapeutic drug monitoring: Quantification of clozapine and norclozapine in human plasma Drug Test Anal. 2018;10:323-329.

17. Mano Y, Asakawa Y, Kita K, Ishii T, Hotta K and Kusano K Validation of an ultraperformance liquid chromatography-tandem mass spectrometry method for the determination of flecainide in human plasma and its clinical application Biomed Chromatogr. 2015;29:1399-1405. 


\section{Table and Figure legends:}

Table 1. Accuracy and precision data: analysis of the IQC solutions using LC-TOF-MS.

Table 2. Summary stability data.

Figure 1. Example chromatograms for an LLoQ calibrator $(100 \mu \mathrm{g} / \mathrm{L})$. Shown are (A) flecainide with, inset, the flecainide- ${ }^{13} \mathrm{C}$ qualifier ion chromatogram, and (B) flecainide- $\mathrm{D}_{4}$. Figure 2. Comparison between LC-MS/MS and LC-TOF-MS results ( $\mathrm{N}=55$ patient samples - solid dots; $N=6$ EQA samples - crosses). Shown are (top) a scatter plot and (bottom) a difference plot of the results produced by both methods. The scatter plot shows the line of identity (solid black line), and the correlation curve (dashed line). The difference plot shows the line of zero bias (solid black line), and the mean ( $\pm 95 \%$ limits of agreement) bias (thick black dashed line and fine black dashed lines, respectively). 
Table 1. Accuracy and precision data: analysis of the IQC solutions using LC-TOF-MS.

\begin{tabular}{|l|c|c|c|c|c|}
\hline Within-assay & IQC A & IQC B & IQC C & LLoQ & ULoQ \\
\hline Flecainide & 200 & 400 & 800 & 100 & 1,500 \\
\hline Nominal $(\mu \mathrm{g} / \mathrm{L})$ & 200.3 & 413.5 & 819.5 & 102.1 & $1,421.2$ \\
\hline Mean measured $(\mu \mathrm{g} / \mathrm{L})$ & 2.83 & 4.09 & 1.12 & 3.11 & 4.64 \\
\hline RSD $(\%)$ & 100.2 & 103.4 & 102.4 & 102.1 & 94.8 \\
\hline Accuracy $(\%)$ & IQC A & IQC B & IQC C & LLoQ & ULoQ \\
\hline Between-assay & 200 & 400 & 800 & 100 & 1,500 \\
\hline Flecainide & 206.5 & 440.2 & 832.9 & 99.5 & $1,442.7$ \\
\hline Nominal $(\mu \mathrm{g} / \mathrm{L})$ & 3.65 & 3.20 & 1.96 & 3.45 & 3.65 \\
\hline Rean measured $(\mu \mathrm{g} / \mathrm{L})$ & 103.2 & 110.0 & 104.1 & 99.5 & 96.2 \\
\hline Accuracy $(\%)$ &
\end{tabular}


Table 2. Summary stability data.

\begin{tabular}{|c|c|c|c|}
\hline \multicolumn{4}{|c|}{ Autosampler stability (post-preparation, $10^{\circ} \mathrm{C}, 48 \mathrm{~h}$ ) } \\
\hline Flecainide & IQC A & IQC B & IQC C \\
\hline Nominal $(\mu \mathrm{g} / \mathrm{L})$ & 200 & 400 & 800 \\
\hline Mean measured $(\mu \mathrm{g} / \mathrm{L})$ & 210 & 445.5 & 834.8 \\
\hline $\operatorname{RSD}(\%)$ & 2.11 & 2.85 & 4.49 \\
\hline Accuracy $(\%)$ & 105.2 & 111.4 & 104.4 \\
\hline \multicolumn{4}{|c|}{ Stability at ambient temperature $\left(15-30^{\circ} \mathrm{C}\right.$, one week $)$} \\
\hline Flecainide & IQC A & IQC B & IQC C \\
\hline Nominal $(\mu \mathrm{g} / \mathrm{L})$ & 200 & 400 & 800 \\
\hline Mean measured $(\mu \mathrm{g} / \mathrm{L})$ & 199.7 & 446.5 & 842.2 \\
\hline $\operatorname{RSD}(\%)$ & 2.22 & 2.01 & 2.09 \\
\hline Accuracy $(\%)$ & 99.9 & 111.6 & 105.3 \\
\hline \multicolumn{4}{|c|}{ Stability stored cooled $\left(2-8^{\circ} \mathrm{C}\right.$, one week $)$} \\
\hline Flecainide & IQC A & IQC B & IQC C \\
\hline Nominal $(\mu \mathrm{g} / \mathrm{L})$ & 200 & 400 & 800 \\
\hline Mean measured $(\mu \mathrm{g} / \mathrm{L})$ & 190.1 & 408.1 & 830.1 \\
\hline $\operatorname{RSD}(\%)$ & 7.97 & 7.61 & 0.57 \\
\hline Accuracy $(\%)$ & 95.1 & 102.0 & 103.8 \\
\hline \multicolumn{4}{|c|}{ Freeze-thaw stability ( 3 x freeze-thaw cycles) } \\
\hline Flecainide & IQC A & IQC B & IQC C \\
\hline Nominal $(\mu \mathrm{g} / \mathrm{L})$ & 200 & 400 & 800 \\
\hline Mean measured $(\mu \mathrm{g} / \mathrm{L})$ & 208.9 & 448.1 & 849.3 \\
\hline $\operatorname{RSD}(\%)$ & 1.28 & 1.33 & 2.01 \\
\hline Accuracy $(\%)$ & 104.5 & 112.0 & 106.1 \\
\hline \multicolumn{4}{|c|}{ Long-term stability $\left(-15\right.$ to $-25^{\circ} \mathrm{C}, 9$ months $)$} \\
\hline Flecainide & IQC A & IQC B & IQC C \\
\hline Nominal $(\mu \mathrm{g} / \mathrm{L})$ & 200 & 400 & 800 \\
\hline Mean measured $(\mu \mathrm{g} / \mathrm{L})$ & 196.9 & 405.8 & 843.0 \\
\hline $\operatorname{RSD}(\%)$ & 5.96 & 7.30 & 8.84 \\
\hline Accuracy (\%) & 98.5 & 101.4 & 105.4 \\
\hline
\end{tabular}


Figure 1. Example chromatograms for an LLoQ calibrator $(100 \mu \mathrm{g} / \mathrm{L})$. Shown are (A) flecainide with, inset, the flecainide- ${ }^{13} \mathrm{C}$ qualifier ion chromatogram, and (B) flecainide- $\mathrm{D}_{4}$.
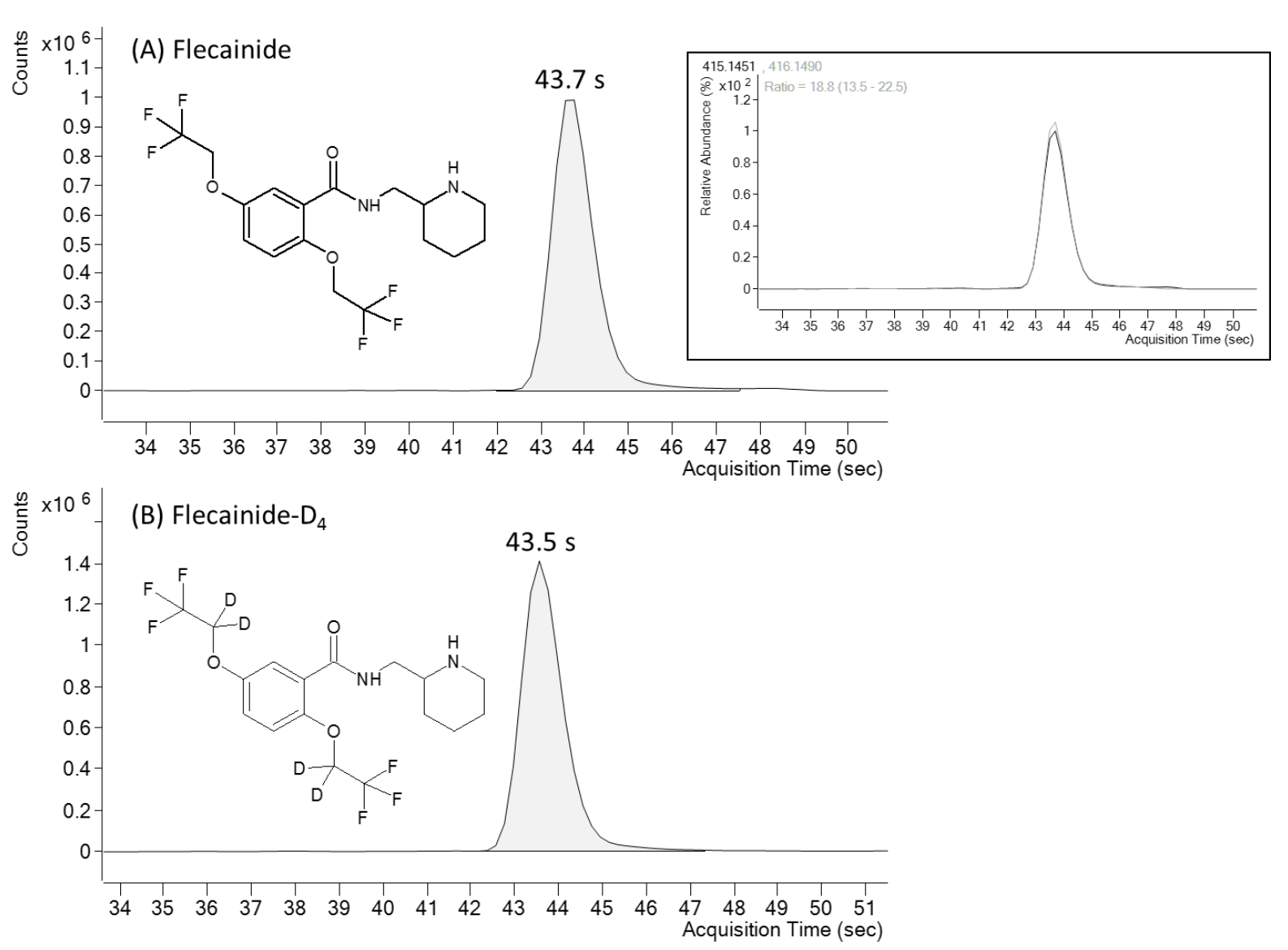
Figure 2. Comparison between LC-MS/MS and LC-TOF-MS results ( $\mathrm{N}=55$ patient samples - solid dots; $\mathrm{N}=6$ EQA samples - crosses). Shown are (top) a scatter plot and (bottom) a difference plot of the results produced by both methods. The scatter plot shows the line of identity (solid black line), and the correlation curve (dashed line). The difference plot shows the line of zero bias (solid black line), and the mean ( $\pm 95 \%$ limits of agreement) bias (thick black dashed line and fine black dashed lines, respectively).
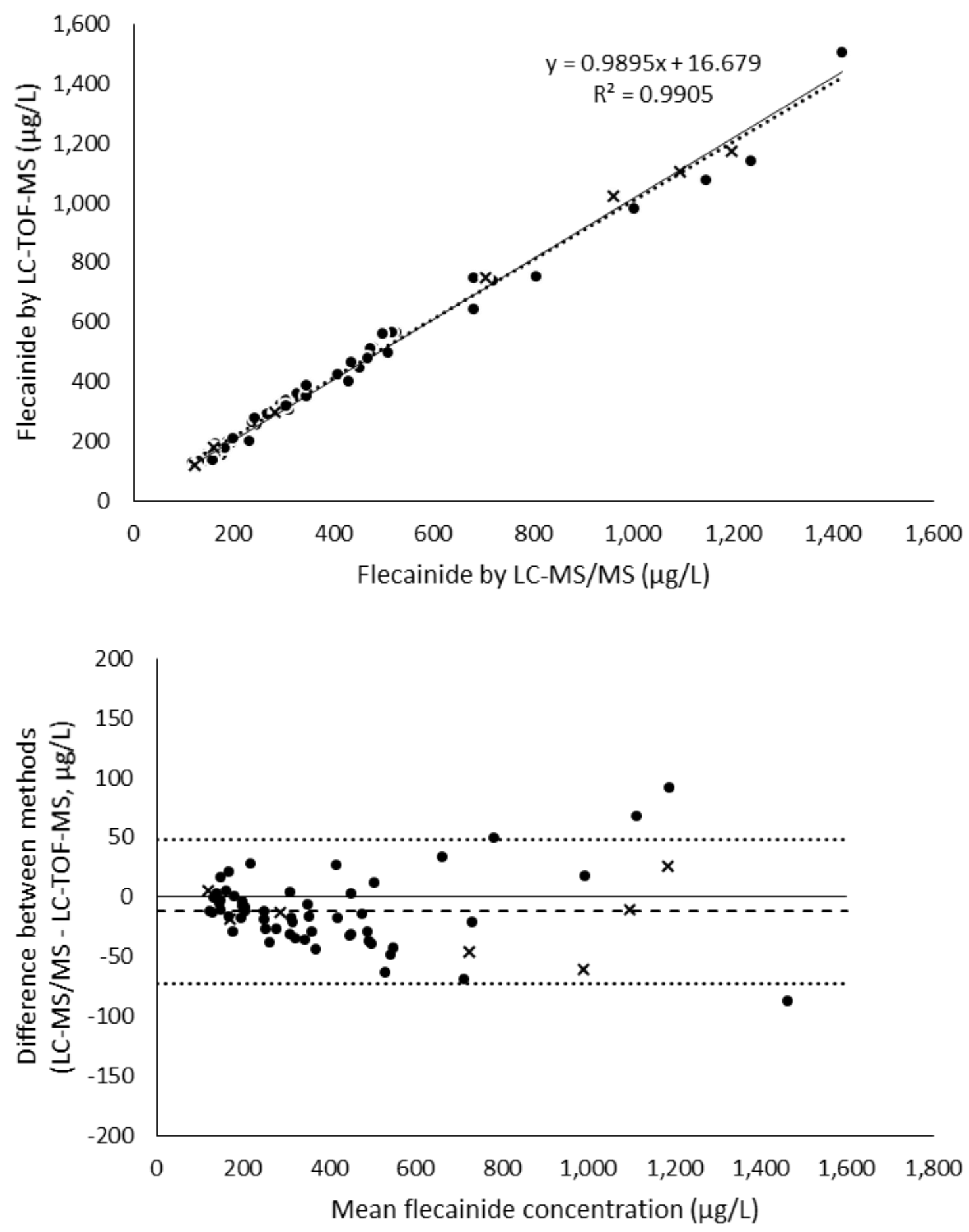


\section{Supplemental Digital Content:}

Supplemental Digital Content 1. LC-MS/MS summary method details.

Supplemental Digital Content 1. docx file

Supplemental Digital Content 2. Summary data from the serial dilution experiment to test assay linearity.

Supplemental Digital Content 2. docx file

Supplemental Digital Content 3. Summary data from five-point mixing experiments to assess the influence of (A) haemolysis and (B) lipaemia.

Supplemental Digital Content 3. docx file 
Supplemental Digital Content 1. Flecainide LC-MS/MS summary method information.

Samples $(50 \mu \mathrm{L})$ were prepared using a two-step liquid-liquid extraction, firstly into methyltert-butyl ether, followed by a back-extraction into aqueous acetic acid. Prepared extracts were injected onto the LC-MS/MS system.

LC-MS/MS was carried out using isocratic elution [300 $\mu \mathrm{L} / \mathrm{min}, 2 \mathrm{mmol} / \mathrm{L}$ ammonium acetate and $0.1 \%$ formic acid in deionised water and methanol $(40+60, v / v)]$ on a reversedphase column $\left(\mathrm{C} 18,50{ }^{\circ} \mathrm{C}\right)$. Flecainide and flecainide- $\mathrm{D}_{4}$ eluted at $2.8 \mathrm{~min}$, and the total analysis time was 5 minutes.

MS/MS source conditions (API2000 mass spectrometer) were: turbo-heater temperature 400 ${ }^{\circ} \mathrm{C}$, curtain gas pressure $20 \mathrm{psig}$, collision gas pressure 6 psig, ionspray voltage $5,500 \mathrm{~V}$. The pre-collision cell voltages were optimised for declustering potential (DP, $61 \mathrm{~V}$ ), focusing potential $(\mathrm{FP}, 370 \mathrm{~V})$, entrance potential $(\mathrm{EP}, 12 \mathrm{~V})$, collision energy $(\mathrm{CE}, 41 \mathrm{~V})$ and collision cell exit potential (CXP, $6 \mathrm{~V})$.

The flecainide/flecainide-D4 precursor ion and product ion were scanned at 415.122/419.140 and 300.900/305.100 respectively. Instrument control and data acquisition were performed using Analyst ${ }^{\mathrm{TM}}$ (version 1.6.3, AB Sciex). 
Supplemental Digital Content 2. Summary data from the serial dilution experiment to test assay linearity.

\begin{tabular}{|c|c|c|c|c|c|}
\hline $\begin{array}{c}\text { Dilution } \\
\text { from } \\
\mathbf{U L o Q} \\
(\boldsymbol{v} / \boldsymbol{v})\end{array}$ & $\begin{array}{c}\text { Mean measured } \\
\text { concentration } \\
(\boldsymbol{\mu} \mathbf{g} / \mathbf{L}, \mathbf{N}=\mathbf{3})\end{array}$ & $\mathbf{S D}(\boldsymbol{\mu g} / \mathbf{L})$ & $\mathbf{R S D}(\boldsymbol{\%})$ & $\begin{array}{c}\text { Calculated } \\
\text { expected) } \\
\text { concentrati } \\
\mathbf{o n}(\boldsymbol{\mu g} / \mathbf{L})\end{array}$ & $\begin{array}{c}\text { Accuracy } \\
(\boldsymbol{\%})\end{array}$ \\
\hline N/A & 1429.7 & 19.82 & 1.39 & 1429.7 & - \\
\hline $1+1$ & 777.5 & 7.47 & 0.96 & 714.9 & 108.8 \\
\hline $1+3$ & 386.4 & 3.53 & 0.91 & 357.4 & 108.1 \\
\hline $1+7$ & 180.2 & 3.04 & 1.69 & 178.7 & 100.8 \\
\hline $1+15$ & 82.45 & 4.16 & 5.04 & 89.4 & 92.3 \\
\hline $1+31$ & 35.10 & 1.30 & 3.71 & 44.7 & 78.5 \\
\hline $1+63$ & 10.82 & 1.41 & 13.06 & 22.3 & 48.4 \\
\hline
\end{tabular}


Supplemental Digital Content 3. Summary data from five-point mixing experiments to assess the influence of (A) haemolysis and (B) lipaemia.

(A) Haemolysis

\begin{tabular}{|c|c|c|c|c|}
\hline Sample ID & $\begin{array}{c}\text { Measured } \\
\text { concentration } \\
(\mu \mathrm{g} / \mathrm{L})\end{array}$ & $\begin{array}{c}\text { Mean } \\
\text { measured } \\
\text { concentration } \\
(\mu \mathrm{g} / \mathrm{L}) \\
\end{array}$ & $\begin{array}{c}\text { Calculated } \\
\text { (expected) } \\
\text { concentration } \\
(\mu \mathrm{g} / \mathrm{L}) \\
\end{array}$ & $\begin{array}{c}\text { Accuracy } \\
(\%)\end{array}$ \\
\hline \multirow{5}{*}{$\begin{array}{c}\text { Neat 'normal' } \\
\text { plasma, } 100 \mu \mathrm{g} / \mathrm{L}\end{array}$} & 101.02 & \multirow{5}{*}{103.01} & \multirow{5}{*}{103.01} & 98.1 \\
\hline & 101.49 & & & 98.5 \\
\hline & 110.90 & & & 107.7 \\
\hline & 101.82 & & & 98.8 \\
\hline & 99.82 & & & 96.9 \\
\hline \multirow{5}{*}{$\begin{array}{c}\text { Mix } \mathbf{1}(3+1, v / v, \\
\text { normal plasma } \\
\text { and haemolysed } \\
\text { plasma })\end{array}$} & 474.16 & \multirow{5}{*}{483.15} & \multirow{5}{*}{476.06} & 99.6 \\
\hline & 479.24 & & & 100.7 \\
\hline & 478.52 & & & 100.5 \\
\hline & 481.27 & & & 101.1 \\
\hline & 502.57 & & & 105.6 \\
\hline \multirow{5}{*}{$\begin{array}{c}\text { Mix } 2(1+1, v / v, \\
\text { normal plasma } \\
\text { and haemolysed } \\
\text { plasma })\end{array}$} & 909.55 & \multirow{5}{*}{913.19} & \multirow{5}{*}{849.11} & 107.1 \\
\hline & 935.81 & & & 110.2 \\
\hline & 906.55 & & & 106.8 \\
\hline & 916.75 & & & 108.0 \\
\hline & 897.31 & & & 105.7 \\
\hline \multirow{5}{*}{$\begin{array}{c}\text { Mix } 3(1+3, v / v, \\
\text { normal plasma } \\
\text { and haemolysed } \\
\text { plasma })\end{array}$} & 1331.65 & \multirow{5}{*}{1281.83} & \multirow{5}{*}{1222.16} & 109.0 \\
\hline & 1340.57 & & & 109.7 \\
\hline & 1249.77 & & & 102.3 \\
\hline & 1246.55 & & & 102.0 \\
\hline & 1240.62 & & & 101.5 \\
\hline \multirow{5}{*}{$\begin{array}{c}\text { Neat haemolysed } \\
\text { plasma, } 1,500 \\
\mu \mathrm{g} / \mathrm{L}\end{array}$} & 1625.53 & \multirow{5}{*}{1595.21} & \multirow{5}{*}{1595.21} & 101.9 \\
\hline & 1587.32 & & & 99.5 \\
\hline & 1609.61 & & & 100.9 \\
\hline & 1565.50 & & & 98.1 \\
\hline & 1588.11 & & & 99.6 \\
\hline
\end{tabular}


(B) Lipaemia

\begin{tabular}{|c|c|c|c|c|}
\hline Sample ID & $\begin{array}{c}\text { Measured } \\
\text { concentration } \\
(\mu \mathrm{g} / \mathrm{L})\end{array}$ & $\begin{array}{c}\text { Mean } \\
\text { measured } \\
\text { concentration } \\
(\mu \mathrm{g} / \mathrm{L}) \\
\end{array}$ & $\begin{array}{c}\text { Calculated } \\
\text { (expected) } \\
\text { concentration } \\
(\mu \mathrm{g} / \mathrm{L}) \\
\end{array}$ & $\begin{array}{c}\text { Accuracy } \\
(\%)\end{array}$ \\
\hline \multirow{5}{*}{$\begin{array}{c}\text { Neat 'normal' } \\
\text { plasma, } 100 \mu \mathrm{g} / \mathrm{L}\end{array}$} & 94.44 & \multirow{5}{*}{94.25} & \multirow{5}{*}{94.25} & 100.2 \\
\hline & 94.50 & & & 100.3 \\
\hline & 93.44 & & & 99.1 \\
\hline & 95.67 & & & 101.5 \\
\hline & 93.22 & & & 98.9 \\
\hline \multirow{5}{*}{$\begin{array}{c}\text { Mix } 1(3+1, v / v, \\
\text { normal plasma } \\
\text { and lipaemic } \\
\text { plasma })\end{array}$} & 450.75 & \multirow{5}{*}{475.63} & \multirow{5}{*}{457.77} & 98.5 \\
\hline & 484.36 & & & 105.8 \\
\hline & 477.33 & & & 104.3 \\
\hline & 483.26 & & & 105.6 \\
\hline & 482.45 & & & 105.4 \\
\hline \multirow{5}{*}{$\begin{array}{c}\text { Mix } 2(1+1, v / v, \\
\text { normal plasma } \\
\text { and lipaemic } \\
\text { plasma })\end{array}$} & 845.18 & \multirow{5}{*}{838.51} & \multirow{5}{*}{868.42} & 97.3 \\
\hline & 843.36 & & & 97.1 \\
\hline & 839.37 & & & 96.7 \\
\hline & 812.99 & & & 93.6 \\
\hline & 851.67 & & & 98.1 \\
\hline \multirow{5}{*}{$\begin{array}{c}\text { Mix } \mathbf{3}(1+3, v / v \\
\text { normal plasma } \\
\text { and lipaemic } \\
\text { plasma })\end{array}$} & 1205.95 & \multirow{5}{*}{1201.42} & \multirow{5}{*}{1184.81} & 101.8 \\
\hline & 1190.83 & & & 100.5 \\
\hline & 1190.47 & & & 100.5 \\
\hline & 1196.31 & & & 101.0 \\
\hline & 1223.57 & & & 103.3 \\
\hline \multirow{5}{*}{$\begin{array}{c}\text { Neat lipaemic } \\
\text { plasma, } 1,500 \\
\mu \mathrm{g} / \mathrm{L}\end{array}$} & 1539.83 & \multirow{5}{*}{1548.33} & \multirow{5}{*}{1548.33} & 99.5 \\
\hline & 1548.39 & & & 100.0 \\
\hline & 1555.15 & & & 100.4 \\
\hline & 1571.83 & & & 101.5 \\
\hline & 1526.44 & & & 98.6 \\
\hline
\end{tabular}

